\title{
Desarrollo de las habilidades del pensamiento en estudiantes de Tercer Año de Bachillerato de la Unidad Educativa "Amelia Gallegos Díaz" - Riobamba
}

\section{Development of the Skills of the Thought in students of Third Year of Baccalaureate of the Educational Unit "Amelia Gallegos Díaz" - Riobamba}

\author{
Maria Belén Piñas Morales ${ }^{1 *}$, Antonio Paredes Peñaherrera ${ }^{1}$ y Tannia Alexandra Casanova Zamora ${ }^{1}$ \\ ${ }^{1}$ Universidad Nacional de Chimborazo \\ *belenpinas@gmail.com
}

DOI: https://doi.org/10.26871/killkana_social.v3i2.462

\begin{abstract}
Resumen
El examen Ser Bachiller es un instrumento que evalúa el desarrollo de las aptitudes y destrezas que los estudiantes que deben alcanzar al culminar la educación intermedia, y que son necesarias para acceder a los estudios de Educación Superior. El objetivo de la investigación es analizar el impacto de las capacitaciones realizadas a los estudiantes de tercer año de bachillerato de las Unidades Educativas de la provincia de Chimborazo, en el área de Ciencias Naturales,especificamente en el desarrollo de las habilidades del razonamiento numérico, abstracto y verbal. Con relación a los puntajes obtenidos en el examen Ser Bachiller, la investigación es de carácter descriptiva y explicativa, utilizando como instrumento un estudio de campo con la aplicación de una prueba de diagnóstico sin capacitación y una evaluación final después de recibir la capacitación. El resultado principal fue que la gran mayoría de estudiantes posee una alta deficiencia en conocimientos con relación a las áreas de razonamiento numérico, Ciencias naturales, razonamiento verbal y abstracto, mientras que un mínimo porcentaje demuestra un mejor nivel de conocimiento.
\end{abstract}

Palabras clave: Habilidades, pensamiento, numérico, abstracto, verbal, Ciencias naturales.

\begin{abstract}
Intelligence and skills are concepts that have a close relationship with each other and are directly linked to the development of human thought and this in turn is analogous to the development of society. This research aims to analyze the educational perspective assumed by the Ecuadorian government regarding the topic "development of thinking skills" in young people who are in the last year of high school, the same one that responds to the pedagogical model implemented in the country called critical pedagogy. the document presents a study based on the results obtained in the ENES tests applied by the Ecuadorian government in the years 2013 and 2014 in high school students. It should be noted that this test is an essential requirement for admission to Ecuadorian public universities. The results clearly reflect a marked difference between the students of the city and the periphery, the urban and rural sector. The results clearly reflect a marked difference between the students of the city and the periphery, the urban and rural sector, and evidence the widening in the existing gap between the different sectors that conform society, the axiology to propose feasible solutions. The data reflect a significant increase in the percentage of students who enter the university, but on the other hand it is evident that the increase is noticeably lower in the fiscal and rural educational institutions. This situation motivated the Universidad Nacional de Chimborazo to design a project called "development of thinking skills in high school students of the schools of the city of Riobamba that seeks to increase the intellectual skills of these sectors through a process of planned and continuous training.
\end{abstract}

Key words: Development, Skills, Thought, Chimborazo.

\section{Introducción}

El Ministerio de Educación emprendió desde el año 2012, el proceso de "reordenamiento de la oferta educativa", lo que generó la disminución del número de institucio- nes educativas. Este proceso de planificación pretende reestructurar las unidades o instituciones que se encuentran en funcionamiento, para lo cual se consideran las necesidades de cada territorio. 
En el año 2007 solo 50 de cada 100 jóvenes asistía a bachillerato en la edad correspondiente. Para el 2014 la tasa neta de asistencia a bachillerato fue de $65,1 \%$, lo que significa que 65 de cada 100 jóvenes en edad de asistir a bachillerato lo están haciendo; esta tasa en comparación con el año 2013 descendió en 0,7\%. («PUB_EstadisticaEducativaVol1_mar2015.pdf», s. f.)

Según la Revista Estadística Educativa (2015) del Ministerio de Educación del Ecuador, el descenso de la tasa neta de asistencia a bachillerato se debe al incremento de las personas que no desean estudiar ya sea por falta de recursos económicos (25,4\% de la población de 15 a 17 años), o porque no están interesados $(20,4 \%$ de la población de 15 a 17 años).

La falta de recursos económicos es un factor importante en los estudiantes de tercer año de bachillerato, para poder acceder a las capacitaciones que ofertan instituciones particulares con el objetivo de prepararles para el examen Ser Bachiller. El proyecto Desarrollo de las Habilidades de Pensamiento en estudiantes de tercer año de bachillerato de las Unidades Educativas de la provincia de Chimborazo en el periodo lectivo 2017-2018, ha sido creado con la finalidad de atender a los estudiantes del sector vulnerable de la provincia de Chimborazo en la capacitación en el área de Ciencias Naturales, razonamiento numérico, abstracto y verbal, previo a la rendición del examen Ser Bachiller, instrumento que evalúa el desarrollo de las aptitudes y destrezas que los estudiantes deben alcanzar al culminar la educación intermedia y que son necesarias para acceder a estudios de educación superior.

La ejecución de dicho proyecto ha permitido determinar con claridad el nivel bajo del Desarrollo de las Habilidades del Pensamiento de los estudiantes principalmente en razonamiento numérico y Ciencias Naturales seguido por razonamiento verbal y finalmente abstracto, considerando como factor principal que los estudiantes proceden de sectores vulnerables, donde se evidencia la falta de recursos económicos y donde muchos de ellos primero cumplen con el trabajo de la agricultura para después realizar las actividades académicas.

\section{Marco teórico}

La fundamentación teórica que apoya al proyecto del desarrollo de las habilidades se basa en el pensamiento de Gardner, (1999) quien manifiesta que:

El objetivo principal de la educación es promover el desarrollo personal de los alumnos, en todas sus capacidades mentales: cognitivas, afectivas, morales y sociales, en la confianza y expectativa optimista de conseguir, además de vidas personales más realizadas, una sociedad cada vez más justa y solidaria. La educación debe proporcionar las bases y 7 recursos para potenciar la comprensión de nuestros diversos mundos: el mundo físico, el mundo biológico, el mundo de las personas, el mundo de las tecnologías, y el mundo personal (Aloso, pág. 6).

\subsection{Aprendizaje significativo}

Según Ausubel, Novak y Hanesian (2009), el aprendizaje significativo ocurre cuando nuevas ideas, conceptos y proposiciones pueden ser aprendidos de manera significativa en la medida en que otros conceptos, ideas o proposiciones relevantes estén claros y disponibles en la estructura cognitiva del individuo. De esta manera el sujeto establece una relación sustantiva entre los conceptos que posee y la nueva información. (Capilla, 2016).

De acuerdo con Pozo (2006) y Rivas (2010) la teoría del aprendizaje significativo además de sustentarse en las teorías psicogenética y sociocultural, también recupera premisas centrales de la teoría del procesamiento de la información retomando la premisa de los procesos cognitivos generales: recopilación de información, conexión entre la nueva información y la existente, recuperación y aplicación de la información. (Capilla, 2016).

El aprendizaje significativo trasciende de la repetición memorística a la aprehensión del conocimiento, dando sentido a lo aprendido y a entender el mundo que lo rodea. Para Ausubel adquirir gran cantidad de volumen de conocimiento es sencillamente imposible si no hay aprendizaje significativo.

El aprendizaje significativo es el proceso según el cual se relaciona un nuevo conocimiento o una nueva información con la estructura cognitiva de la persona que aprende de forma no arbitraria y sustantiva o no literal. Esa interacción con la estructura cognitiva no se produce considerándola como un todo, sino con aspectos relevantes presentes en la misma, que reciben el nombre de subsumidores o ideas de anclaje (Ausubel, 1976, 2002; Moreira, 1997a). La presencia de ideas, conceptos o proposiciones inclusivas, claras y disponibles en la mente del aprendiz es lo que dota de significado a ese nuevo contenido en interacción con el mismo (Moreira, 2000a). Esa interacción es lo que caracteriza al aprendizaje significativo. Citado en (Capilla, 2016).

Ausubel distingue tres tipos fundamentales de aprendizaje significativo (Aceituno,1998):

- Aprendizaje representacional: tipo básico de aprendizaje significativo. En él se asignan significados a determinados símbolos (palabras) se identifican los símbolos con sus referentes (objetos, eventos, conceptos).

- Aprendizaje de conceptos: los conceptos representan regularidades de eventos u objetos, y son representados también por símbolos particulares o categorías y representan abstracciones de atributos esenciales de los referentes.

- Aprendizaje proposicional: la tarea no es aprender significativamente lo que representan las palabras aisladas o combinadas sino aprender lo que significan las ideas expresadas en una proposición, las cuales a su vez constituyen un concepto. En este tipo de aprendizaje 
la tarea no es aprender un significado aislado de los diferentes conceptos que constituyen una proposición, sino el significados de ella como un todo.

\subsection{Habilidades del pensamiento}

Las habilidades se han considerado como las destrezas de que dispone una persona para aprender, es decir, cómo capta la información que ve, lee y oye; qué hace para procesar esa información; cómo la guarda en la memoria; y cómo la utiliza para razonar y resolver problemas (Habilmind 2012). Citado en (Faria Junior, 2015).

Las habilidades del pensamiento están directamente relacionada con la cognición, que incluye diferentes formas de conocer algo, se relaciona también con otros procesos como la percepción, la memoria, aprendizaje y el razonamiento, siendo este último la habilidad más importante del pensamiento. Las habilidades del pensamiento están relacionadas entre sí, no se pueden identificar por sí solas, por ejemplo para sintetizar es necesario el análisis o la inferencia.

Manzano (1988) ha propuesto 21 operaciones cognitivas agrupadas en ocho habilidades del pensamiento básico: habilidades de enfoque (definir problemas y establecer metas), habilidades para recopilar la información (observar y formular preguntas), habilidades de memoria (almacenar y recordar), habilidades de análisis (identificar atributos y componentes, identificar relaciones y patrones, identificar relaciones y patrones, identificar ideas principales, identificar errores), habilidades de construcción (inferir, predecir, elaborar), habilidades de evaluación (establecer criterios).

Para determinar qué habilidades son fundamentales para el desarrollo del pensamiento crítico, debe partirse del conocimiento de lo que significa el pensamiento. Una definición de pensamiento muy sintetizada es la que propone Ennis (1997). Para este autor, la expresión "pensamiento crítico" generalmente es utilizada cuando se quiere destacar una actividad práctica reflexiva, que tiene como objetivo una creencia o una acción sensata. De esta forma, el pensamiento crítico puede definirse como una forma de pensamiento racional, reflexivo, centrado en lo que se quiere creer o hacer (Faria Junior, 2015).

\section{Metodología}

El presente estudio utiliza un nivel de investigación descriptivo que muestra las características del fenómeno estudiado. Es además explicativa porque busca responder a la problemática de marcadas deficiencias de los estudiantes en el desarrollo de los distintos razonamientos, presenta un análisis cuantitativo que se sustenta en la aplicación de instrumentos de evaluación que miden el nivel de desarrollo de las habilidades del pensamiento de los estudiantes comparado entre una prueba diagnóstico sin capacitación, con una prueba final una vez concluido un proceso de capacitación de 100 horas en las modalidades presencial y virtual.
El problema identificado se describe como "el bajo índice de estudiantes de colegios públicos, que aprueban el examen para el ingreso a las universidades del país."

Se delimita el objetivo general del estudio, que es investigar el nivel de desarrollo de las habilidades de pensamiento abstracto, numérico y verbal y el área de Ciencias Naturales y Biología Química.

La muestra utilizada está constituida por estudiantes de tercer año de bachillerato de los la unidad educativa Amelia Gallegos Díaz de la ciudad de Riobamba, provincia de Chimborazo, períodos académicos 2016 - 2018. El estudio no considera el sexo ya que esta variable no se supone determinante para el presente estudio.

La población objeto de investigación, es de 171, y está constituida por estudiantes de tercer año de bachillerato de la Unidad Educativa antes mencionada. El muestreo es intencional, ya que depende de la decisión particular de cada estudiante para inscribirse en talleres de capacitación ofertados por la UNACH. La muestra está constituida por los estudiantes inscritos en las capacitaciones ofertadas mediante el proyecto de vinculación e incluye a todos los estudiantes inscritos.

Los estudiantes investigados reciben 10 jornadas de capacitación de seis horas cada una en cuatro razonamientos: verbal, numérico, abstracto y Ciencias Naturas y Biología, durante los días sábados. En una primera jornada se aplicó una prueba diagnóstica que midió los conocimientos previos, al finalizar en la última jornada, es decir en la décima se aplicó una segunda prueba denominada final que permitió comparar los resultados alcanzados y medir el nivel de avance de los estudiantes intervenidos.

Las pruebas contienen un total de ochenta preguntas de las cuales 20 corresponden a cada razonamiento, que es aplicada en un tiempo de 120 minutos, lo que corresponde a 1,50 minutos de tiempo para responder cada pregunta.

El análisis de los informes se sustenta en cuatro variables de estudio: habilidades numéricas, abstractas, verbales y ciencias naturales, cuatro indicadores por instrumento: Dominio Matemático: resolución de problemas estructurados, relaciones entre variables y sus representaciones, razones y proporciones; Dominio Abstracto: organización y análisis de información, relaciones y patrones; Dominio Lingüístico: comprensión de textos escritos, elementos de la lengua, semántica contextual, pensamiento analógico verbal; Dominio Científico: elementos y procesos del ecosistema, vínculos entre los mecanismos físico-químicos y la conservación de los recursos naturales, interacción de los sistemas de vida, dinámicas entre materia y energía.

Uno de los instrumentos utilizados fue la Guía de estudios con contenidos teórico-práctico de razonamiento verbal, numérico y abstracto y Ciencias Naturales que fue elaborado por docentes del proyecto de vinculación. A fin de incrementar la fiabilidad de la medición se propuso realizar pruebas semanales para ejercitar a los estudiantes paulatinamente hasta concluir con la prueba final y alcanzar un valor de 46,75\% como mínimo; lo cual indica que 
se dio un avance en el desarrollo de las habilidades del pensamiento de los estudiantes, considerando que al iniciar en la prueba de diagnóstico alcanzaron un promedio de $30,30 \%$.

\section{Resultados}

De los resultados del informe Ser Bachiller del año lectivo 2017-2018 se obtiene los siguientes resultados: numérico $48 \%$, verbal, 57,5\%, abstracto $63 \%$, Ciencias Naturales $48 \%$, lo que determina que la capacitación realizada a través del proyecto de vinculación fortalece las habilidades del pensamiento en los estudiantes intervenidos.

Los resultados han sido agrupados aplicando instrumentos a toda la población de estudiantes del Tercer Año de Bachillerato del período académico 2017 - 2018, pertenecientes a la Unidad Educativa Amelia Gallegos Díaz de la ciudad de Riobamba, Provincia de Chimborazo y comparados con los obtenidos en el Informe de Resultados Ser Bachiller de idéntico año. El análisis utilizado permitió examinar las variables sobre pensamiento verbal, numérico y abstracto y Ciencias Naturales. Se añade además una comparación entre resultados obtenidos entre los años 2016-2017 y 2017-2018 para evidenciar los avances con proyecto (con capacitación) y sin proyecto (sin capacitación).

Se exponen a continuación los resultados correspondientes a cada una de ellas.

Tabla 1 Resultados pre y post evaluación

\begin{tabular}{|c|c|c|c|}
\hline $\begin{array}{c}\text { VARIABLES DE } \\
\text { ESTUDIO }\end{array}$ & $\begin{array}{c}\text { PRUEBA } \\
\text { DIAGNÓSTICO }\end{array}$ & $\begin{array}{c}\text { PRUEBA } \\
\text { FINAL }\end{array}$ & $\begin{array}{c}\text { INFORME SER } \\
\text { BACHILLER }\end{array}$ \\
\hline NUMÉRICO & $25,53 \%$ & 44 & 48 \\
\hline ABSTRACTO & $37 \%$ & 61 & 63 \\
\hline VERBAL & $34 \%$ & 49 & 57,5 \\
\hline CCNN & $25 \%$ & 33 & 48 \\
\hline TOTAL & $30,38^{\circ}$ & 46,75 & 54,13 \\
\hline
\end{tabular}

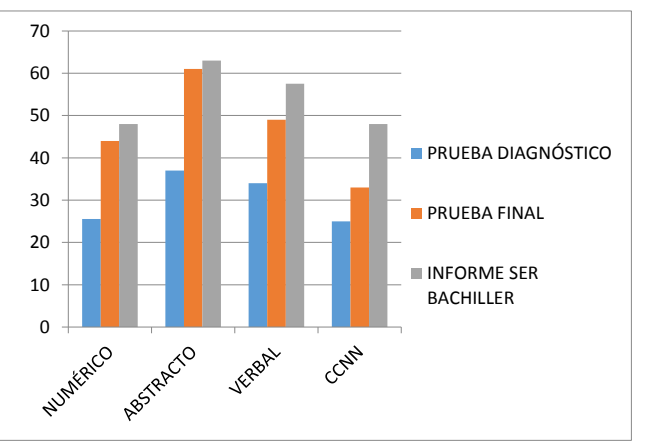

Figura 1.

Los resultados como se muestra en la tabla 1 corresponden a la Evaluación diagnóstica, final e informe Ser Bachiller en cuatro variables de análisis, en el siguiente orden: numérico, abstracto, verbal y Ciencias Naturales. Se evidencia el porcentaje de aciertos de la prueba diagnóstica cuyo resultado es: numérico $25,53 \%$; abstracto $37 \%$; verbal $34 \%$ y Ciencias Naturales $25 \%$ con un total de $30,38 \%$.
Se observa que el mayor nivel de aciertos corresponde a abstracto seguido de verbal, numérico y Ciencias Naturales; estos dos últimos valores muy semejantes logrando un promedio total de aciertos de 30,38\%

En la prueba final los resultados son: numérico $44 \%$, abstracto $61 \%$, verbal $49 \%$ y Ciencias Naturales $33 \%$. Se observa que el mayor nivel de aciertos corresponde a abstracto seguido de verbal, numérico y finalmente Ciencias Naturales que coincide en su totalidad con la prueba diagnóstico y se logra un promedio total de 46,75\%.

En el Informe Ser Bachiller, los resultados son: numérico $48 \%$, abstracto $63 \%$, verbal $57,5 \%$, Ciencias Naturales $48 \%$. Se observa que el mayor nivel de aciertos corresponde a abstracto, seguido de verbal, numérico y Ciencias Naturales estos dos últimos con el mismo valor. Se logra un promedio total de 54,13\%.

La tendencia en las tres pruebas es coincidente manteniendo a razonamiento abstracto con el mayor nivel de aciertos, seguido de verbal y luego, Ciencias Naturales y numérico se mantienen en el último con similar porcentaje.

La prueba en la que se alcanza los mayores porcentajes es Ser Bachiller, seguido de la prueba Final y en último lugar la prueba Diagnóstico. El comportamiento es lógico en el tiempo de aplicación de las pruebas, de modo ascendente, notándose un avance secuencial significativo durante el proceso de capacitación. Sin embargo, el nivel de aciertos alcanzado en la Prueba Ser Bachiller (la más alta), es insuficiente para acceder con facilidad a un cupo en las universidades del país.

Tabla 2 Prueba ser bachiller comparación entre los años 2016-2017 y 2017-2018

\begin{tabular}{lcc}
\hline NUMÉRICO & $2016-2017$ & $2017-2018$ \\
\hline $\begin{array}{l}\text { Resolución de problemas } \\
\text { estructurados }\end{array}$ & $49 \%$ & $49 \%$ \\
$\begin{array}{l}\text { Relaciones entre variables y } \\
\text { sus representaciones }\end{array}$ & $40 \%$ & $52 \%$ \\
Razones y proporciones & $39 \%$ & $43 \%$ \\
\hline
\end{tabular}

Fuente: Instituto Nacional de Evaluación Educativa, 2018

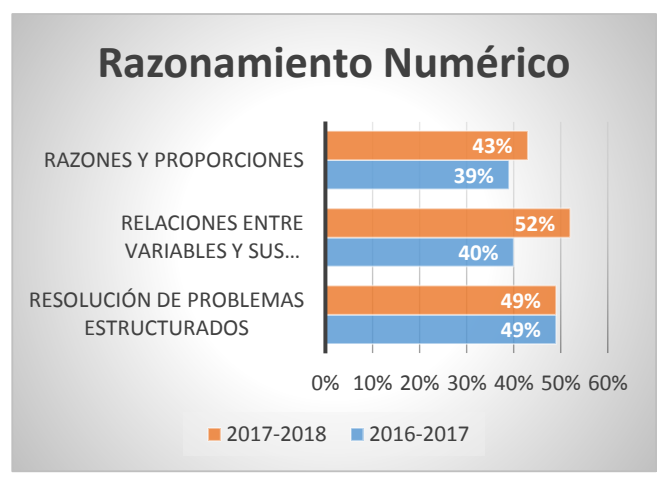

Figura 2. 
En la variable Razonamiento Numérico, se observa un avance respecto a los años comparados en los campos razones y proporciones y relaciones entre variables; en cuanto al campo resolución de problemas estructurados los resultados son coincidentes.

Tabla 3

\begin{tabular}{lcc}
\hline ABSTRACTO & $\mathbf{2 0 1 6 - 2 0 1 7}$ & $\mathbf{2 0 1 7 - 2 0 1 8}$ \\
\hline $\begin{array}{l}\text { Relaciones y patrones } \\
\text { Organización y análisis de } \\
\text { información }\end{array}$ & $64 \%$ & $72 \%$ \\
\hline
\end{tabular}

Fuente: Instituto Nacional de Evaluación Educativa, 2018

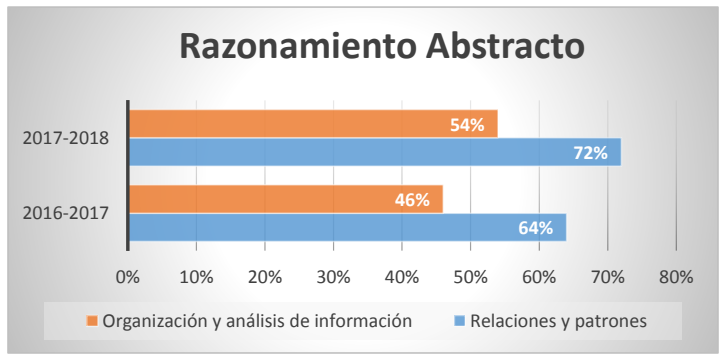

Figura 3.

En la variable Razonamiento Abstracto, se observa un avance respecto a los años comparados en los campos: organización y análisis de información y, relaciones y patrones, notándose mejores resultados en el segundo campo.

Tabla 4

\begin{tabular}{lcc}
\hline VERBAL & $\mathbf{2 0 1 6 - 2 0 1 7}$ & $\mathbf{2 0 1 7 - 2 0 1 8}$ \\
\hline Comprensión de textos & & \\
escritos & $53 \%$ & $58 \%$ \\
$\begin{array}{l}\text { Elementos de la lengua } \\
\text { Semántica Contextual }\end{array}$ & $51 \%$ & $67 \%$ \\
$\begin{array}{l}\text { Pensamiento Analógico } \\
\text { verbal }\end{array}$ & $42 \%$ & $51 \%$ \\
\hline
\end{tabular}

Fuente: Instituto Nacional de Evaluación Educativa, 2018

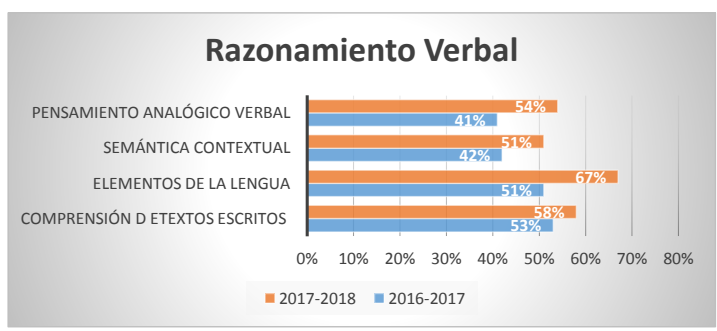

Figura 4.

En la variable Razonamiento Verbal, se observa un avance respecto a los años comparados en todos los campos de análisis, notándose el mayor logro en el campo elementos de la lengua $67 \%$ comparado con $51 \%$ anterior

Tabla 5

\begin{tabular}{lcc}
\hline CCNN & $\mathbf{2 0 1 6 - 2 0 1 7}$ & $\mathbf{2 0 1 7 - 2 0 1 8}$ \\
\hline Elementos y procesos del ecosistema & $49 \%$ & $46 \%$ \\
Vínculos entre los mecanismos físicos químicos & & \\
y la conservación de los recursos naturales & $42 \%$ & $54 \%$ \\
Interacción de los sistemas de vida & $44 \%$ & $51 \%$ \\
Dinámicas entre materia y energía & $30 \%$ & $43 \%$ \\
\hline
\end{tabular}

Fuente: Instituto Nacional de Evaluación Educativa, 2018

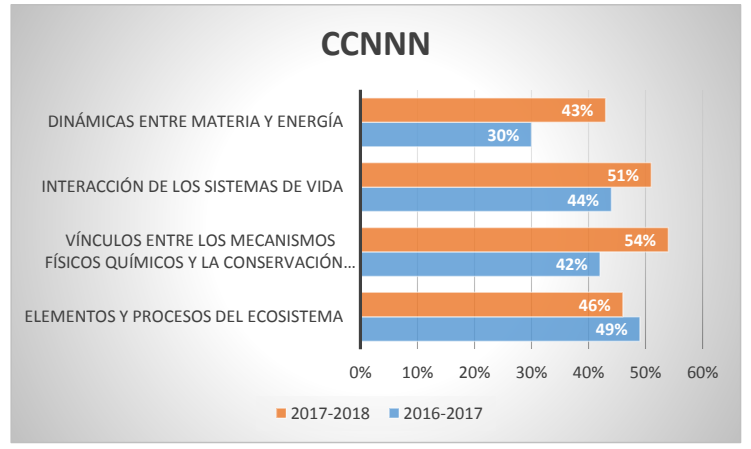

Figura 5.

En la variable Ciencias Naturales, se observa un avance respecto a los años comparados en los campos Dinámicas entre materia y energía, Interacción de los sistemas de vida, Vínculos entre los mecanismos fisico-químicos y la conservación de los recursos naturales; en cuanto al campo Elementos y Procesos del Ecosistema los resultados muestran retroceso aunque en menor escala.

Tabla 6

\begin{tabular}{lrrr}
\hline \multicolumn{1}{c}{ ANÁLISIS ESTAdÍSTICO } & \multicolumn{1}{c}{ PRUEBA } \\
DIAGNÓSTICO & \multicolumn{1}{c}{ PRUEBA FINAL } & $\begin{array}{c}\text { INFORME SER } \\
\text { BACHILLER }\end{array}$ \\
\hline Media & 31 & 47 & 54 \\
Error típico & 2,96 & 5,81 & 3,75 \\
Mediana & 30 & 47 & 53 \\
Moda & $\# N / A$ & $\# N / A$ & 48 \\
Desviación estándar & 5,92 & 11,6 & 7,5 \\
Coeficiente de & & & 13,82 \\
variación & 19,40 & 24,85 & 56 \\
Varianza de la muestra & 35 & 135 & 56 \\
muestra & 35 & 135 & $-3,901,234,568$ \\
Curtosis & $-44,628,571,429$ & 0,6119441 & 0,37037037 \\
Coeficiente de asimetría & 0,193178115 & 0,12108306 & 15 \\
Rango & 12 & 28 & 63 \\
Mínimo & 25 & 33 & 217 \\
Máximo & 37 & 61 & 4 \\
Suma & 122 & 187 & 4 \\
Número de variables & 4 & 4 & \\
\hline
\end{tabular}

\begin{tabular}{|c|c|c|c|c|c|}
\hline & & & HISTOGR & & \\
\hline 2 & 1 & $8,33 \%$ & $\begin{array}{l}\mathrm{y} \\
\text { mayor... }\end{array}$ & 6 & $50,00 \%$ \\
\hline 4 & 5 & $50,00 \%$ & 44 & 5 & $91,67 \%$ \\
\hline $\begin{array}{l}\text { y } \\
\text { mayor... }\end{array}$ & 6 & $100,00 \%$ & 25 & 1 & $100,00 \%$ \\
\hline
\end{tabular}




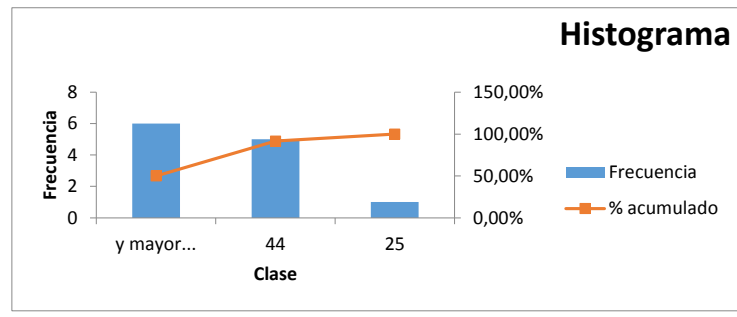

Figura 6.

\section{Conclusiones}

Las siguientes conclusiones que se pueden extraer de este estudio confirman el nivel bajo de desarrollo de Habilidades del Pensamiento de los estudiantes de Tercer Año de Bachillerato de la Unidad Educativa Amelia Gallegos Díaz. Este trabajo da cuenta de una marcada deficiencia en conocimientos y habilidades cognitivas de los estudiantes investigados. La presente investigación ha permitido determinar con claridad la mayor deficiencia en razonamiento numérico y Ciencias Naturales seguido por razonamiento verbal y finalmente razonamiento abstracto

Este estudio ha demostrado que existe desigualdad en los procesos de evaluación y acreditación para la Educación Superior, dado que los estudiantes de Instituciones Educativas Públicas proceden de sectores vulnerables donde se evidencia la falta de recursos económicos, apoyo de sus familias, motivación personal, entre otros; mientras que en otros contextos sociales existen estudiantes adecuadamente preparados y disponen de facilidades para prepararse de mejor forma.

Este hecho determina un alto grado de inequidad en el ingreso a la Educación Superior que cuestiona la validez del sistema de ingreso y la necesidad de generar espacios de debate de la temática en cuestión.

El presente estudio proporciona evidencia adicional para nuevas investigaciones, los nuevos resultados se suman al creciente cuerpo de literatura sobre los procesos educativos en estudiantes bachilleres y su acceso a la Educación Superior ha proveído soporte definitivo a resultados locales y ha proporcionado validación empírica de los mismos.

Los resultados aquí presentados pueden ser también aplicados a otros contextos regionales, nacionales e internacionales, ya que pueden ser comparados para una mayor comprensión del problema y futuras investigaciones.

Se reconocen las limitaciones presentadas durante el proceso de investigación, la mayor, la asistencia irregular de los estudiantes.

El tema ofrece un amplio campo de estudio, que queda por dilucidar y motiva a plantearse nuevas hipótesis de investigación.

\section{Recomendaciones}

Generar desde las universidades, la socialización de experiencias relacionadas que permitan fortalecer los procesos de vinculación e investigación de manera conjunta, y generar proyectos conjuntos entre universidades que permitan optimizar recursos y mejorar la calidad de los servicios entregados.

Generar espacios de capacitaciones desde las propias instituciones educativas y promover convenios interinstitucionales para mejorar el desarrollo de las habilidades del pensamiento en los estudiantes no solo para enfrentarse a la prueba. Estos procesos de capacitación deberían incluir estudiantes desde Educación Básica Inicial, hasta el Bachillerato, es decir, considerar a toda la unidad educativa.

\section{Referencias Bibliográficas}

Capilla, R. M. (2016). Habilidades cognitivas y aprendizaje significativo de la adición y sustracción de fracciones comunes. Cuadernos de Investigación Educativa, 7(2), 49-62.

Díaz Barriga Arceo, F. (2006). Cognición situada $y$ estrategias para el aprendizaje significativo. Descargado de http://ebookcentral . proquest. com/lib/unachlibsp/ detail.action?docID=3169351

Faria Junior, J. B. (2015). Desarrollo de habilidades para contribuir al pensamiento crítico de los estudiantes en la educación de jóvenes y adultos (eja), a través del proceso de enseñanza aprendizaje de la historia de brasil. La Habana, Cuba: Editorial Universitaria. Descargado de http://ebookcentral.proquest.com/ lib/unachlibsp/detail.action?docID= 4775824

Recibido: 3 de mayp de 2019 